\title{
La enseñanza del conflicto y la convivencia en la educación secundaria
}

The Teaching of Conflict and Social Coexistence in High School L'enseignement du conflit et le vivre-ensemble dans l'Education Secondaire

O ensino do conflito e a convivência na educação secundária

Fecha de recepción: 17 DE JUNIO DE 2016/Fecha de aceptación: 25 DE JULIO DE 2017/Fecha de disponibilidad en línea: 1 DE DICIEMBRE DE 2017 Encuentre este artículo en http://magisinvestigacioneducacion.javeriana.edu.co/

Francisco F. García-Pérez

UNIVERSIDAD DE SEVILLA

Sevilla, España

ffgarcia@us.es

\section{Resumen}

En el desarrollo de un proyecto de enseñanza de las Ciencias Sociales en un aula de $4^{\circ}$ de Educación Secundaria Obligatoria en un centro escolar de Sevilla, se investiga la evolución de las concepciones de los alumnos y se constata una progresión en los aprendizajes desde formulaciones más simples del conocimiento hacia concepciones más complejas. Se concluye que la metodología didáctica utilizada, el trabajo en torno a problemas sociales, en distintas escalas, tiene una influencia decisiva en esta evolución.

\section{Palabras clave}

Enseñanza secundaria; educación ciudadana; problema

social; coexistencia pacífica; aprendizaje

Para citar este artículo / To cite this article / Pour citer cet article / Para citar este artigo

Pineda-Alfonso, José Antonio \& García-Pérez, Francisco F. (2017). La enseñanza del conflicto y la convivencia en la educación secundaria. magis, Revista Internacional de Investigación en Educación, 10 (20), 143-158. https://doi.org/10.11144/Javeriana.m10-20.cfse 


\section{Keywords}

Secondary education; citizenship education; social problem; peaceful coexistence; learning

\section{Abstract}

This article presents the development of a social science teaching project in a $4^{\text {th }}$ grade classroom of a high school in Seville. We examined the evolution of students' conceptions and found that there is a progression in their learning from simpler formulations of knowledge to more complex conceptions. It is concluded that the didactic methodology used, the work around social problems, at different scales, has a decisive influence on this evolution.

\section{Mots Clés}

Enseignement secondaire; éducation citoyenne; problème sociale; coexistence pacifique; apprentissage.

\section{Résumé}

Cet article de recherche présente le développement d'un projet d'enseignement des Sciences Sociales à l'intérieure de la salle de classe de $4^{\text {ème }}$ d'Education Secondaire Obligatoire dans un centre scolaire de Sevilla. On a fait la recherche de l'évolution des conceptions des élèves et on a constaté qu'il y a une progression dans les apprentissages depuis les formulations les plus simples de la connaissance envers les conceptions les plus complexes. On conclut que la méthodologie didactique utilisée, le travail tout autour des problèmes sociales, dans les différentes échelles, a une influence décisive sur dite évolution.

\section{Palavras-chave}

Ensino secundário; educação cidadã; problema social; coexistência pacífica; aprendizagem

\section{Resumo}

Este artigo de pesquisa apresenta o desenvolvimento de um projeto de ensino das ciências sociais em uma sala de $4^{\circ}$ ano de educação secundária obrigatória em um centro escolar de Sevilla. Pesquisou-se a evolução das concepções dos alunos e constatamos que se dá uma progressão nas aprendizagens desde formulações mais simples do conhecimento até concepções mais complexas. Conclui-se que a metodologia didática utilizada, o trabalho em torno de problemas sociais, em diferentes escalas, tem uma influência decisiva em dita evolução. 


\section{Introducción: conocimiento escolar y aprendizaje}

Desde las investigaciones pioneras en el campo de la psicología del desarrollo se han sucedido diferentes enfoques teóricos que han aportado evidencias sobre los procesos cognitivos y sus implicaciones para el aprendizaje. Ahora bien, a pesar de que la teoría psicogenética y las investigaciones psicológicas han proporcionado una fundamentación científica de los procesos de enseñanza-aprendizaje, no siempre ha habido una transferencia a las prácticas escolares

En el marco de la teoría constructivista, desde los años 80 del siglo XX, el enfoque del conceptual change ya puso de manifiesto que el aprendizaje no suele ser acumulativo, sino que requiere reconstrucción. Desde esta perspectiva, el cambio se entiende como un proceso temporal, complejo y dinámico y se procura dar cuenta de los mecanismos que lo explican y que dan lugar a las transformaciones psíquicas (Overton, 2013). Posteriormente, el marco clásico del conceptual change ha sido revisado y se han incluido aportaciones diversas, como el papel de las interacciones entre factores cognitivos y emocionales o las ideas del campo de las conceptual metaphors. De hecho, las analogías y las metáforas se consideran principios fundamentales del pensamiento y la acción cotidianos, y se han utilizado como herramienta didáctica, en conexión con las denominadas "experiencias encarnadas" (embodiment), para aclarar aspectos del contenido científico en la práctica del aula (Treagust \& Duit, 2015).

En el ámbito del "conocimiento de lo social" (social cognition), desde las investigaciones pioneras acerca de las ideas infantiles sobre el mundo político, se han sucedido diversos estudios sobre su comprensión por parte de niños y adolescentes [véanse, por ejemplo, las revisiones de Enesco, Delval, Navarro, Villuendas, Sierra \& Peñaranda, 1995; y las más recientes de Álvarez (2012)]. Ahora bien, la social cognition presenta algunas peculiaridades, dado que la psicología del desarrollo ha destacado que la naturaleza psicológica de los individuos no puede disociarse del contexto social en el que tienen lugar el pensamiento, la conducta o las emociones.

De muchas de estas investigaciones se desprende, además, que los contenidos de ciencias sociales que contemplan los currículos se hallan muy alejados de las concepciones de los escolares, al incluir conceptos difíciles de entender por ellos. Por lo demás, este hecho se pone de manifiesto en las encuestas realizadas por la IEA (International Association for the Evaluation of Educational Achievement), que señalan la insuficiencia de conocimientos cívicos e institucionales en cuestiones de ciudadanía y democracia entre los jóvenes de diversos países (Torney-Purta, Lehmann, Oswald \& Schulz, 2001). Todo ello pone de relieve la necesidad de vincular la investigación psicológica a la investigación de propuestas didácticas dirigidas a mejorar la comprensión de la organización social y política de las sociedades (Misco, 2009).

En el campo de la didáctica de las ciencias sociales, algunos de los autores de estos estudios reclaman, así mismo, la necesidad de describir los obstáculos epistémicos que encuentran los niños y adolescentes para comprender las instituciones políticas y los fenómenos sociales (Barrett \& Buchanan-Barrow, 2002). Esto hace necesaria una mayor cooperación entre psicólogos, didactas y educadores en general (Lenzi, Borzi, Pataro \& Iglesias, 2005), para ajustar las propuestas educativas a las necesidades y ritmos de aprendizaje de los alumnos.

En el contexto de la escuela se dan distintos tipos de conocimiento, lo que ha llevado a algunos autores a hablar de "polifasia cognitiva" (Moscovici, 2003), y a otros de "inteligencia cultural", en el sentido de la
Descripción del artículo | Article description | Description de l'article | Artigo descrição

Este artículo de investigación es resultado parcial del proyecto Estrategias de formación del profesorado para educar en la participación ciudadana, con referencia EDU2011-23213, financiado por el Ministerio de Ciencia e Innovación del Gobierno de España y por el Fondo Europeo de Desarrollo Regional, FEDER, desarrollado entre 2012 y 2014. José Antonio Pineda-Alfonso ha desarrollado el proceso de investigación que se presenta en este artículo, con la asesoría y participación de Francisco F. GarcíaPérez, coordinador del proyecto citado, en el cual se enmarca este estudio. 
necesidad de diversificar las inteligencias presentes en los centros escolares. Entre estos conocimientos estarían los disciplinares, las conceptualizaciones construidas por los alumnos, el trasfondo ideológico referido a creencias básicas sobre la sociedad, el conocimiento cotidiano, etc. (LobatoJunior, 2013). Con todos ellos es preciso trabajar en las situaciones de aula.

El conocimiento cotidiano es con frecuencia simplificador, estereotipado y lleno de prejuicios; pero no ayudaremos a los alumnos a construir un conocimiento alternativo sólido si lo que les ofrecemos como conocimiento deseable no es susceptible de conectar con esos conocimientos cotidianos, que son los que, de hecho, les permiten desenvolverse en su mundo. Las disciplinas escolares, por tanto, no son la meta referencial para la construcción del conocimiento, por más que se haya consolidado una larga tradición de presencia de esas materias en el currículum. Por el contrario, la meta es la construcción gradual en el contexto escolar de un tipo de conocimiento general susceptible de ser aplicado a distintos contextos, conocimiento que, a su vez, orienta y da un nuevo sentido a todos los otros conocimientos, más contextuales, manejados por los alumnos (García-Díaz, 1998; García-Pérez, 2000).

Teniendo esto en cuenta, consideramos posible establecer una "hipótesis de progresión" en la comprensión de los contenidos sociales (en relación con lo social, Lenzi, Borzi, Pataro \& Iglesias, 2005, entre otros), de forma que ese camino hipotético constituya una referencia general, tanto para interpretar — desde la perspectiva del aprendizaje — las ideas de los alumnos sobre diversas temáticas, como para programar — desde la perspectiva de la enseñanza - la docencia con una gradualidad en el tratamiento de los contenidos. Ello permite un mejor ajuste a las posibilidades de quienes aprenden, para lograr el enriquecimiento progresivo de su conocimiento (García-Díaz, 1998; García-Pérez, 2000).

Por consiguiente, para conocer esas ideas o concepciones del alumnado, en nuestro proceso investigador elaboramos un sistema de categorías generales que, además de ser utilizado para el análisis de la información en esta investigación, funciona, en el ámbito didáctico, como un andamiaje capaz de estructurar la comprensión de los fenómenos sociales, al propiciar un enriquecimiento y complejización del conocimiento de los alumnos. En la investigación que aquí se presenta nos ha interesado indagar especialmente en la dimensión conceptual de estas concepciones; y con esa perspectiva se ha elaborado el citado sistema de categorías.

\section{Método}

El proyecto de enseñanza objeto de investigación se elaboró en forma de ámbito de investigación escolar (AIE) para la enseñanza de las Ciencias Sociales. Desde la perspectiva del proyecto IRES (Investigación y Renovación Escolar) - que tomamos como marco de referencia- los ámbitos de investigación escolar son conjuntos de problemas socioambientales que actúan como organizadores del currículo y son relevantes para la comprensión de la realidad e integran, definen y concretan el conocimiento escolar deseable (García-Pérez, 2000). En el caso de este AlE se seleccionaron y organizaron los contenidos en torno a problemáticas relacionadas con el "conflicto y la convivencia" (Pineda-Alfonso, 2011, 2013; PinedaAlfonso \& García-Pérez, 2011) y se experimentó en el curso 2010-11 con un grupo de alumnos de $4^{\circ}$ de ESO $(n=21)$ de un centro escolar de educación secundaria en el extrarradio de la ciudad de Sevilla, en una zona socialmente deprimida. A partir de aquí, nos planteamos dar respuesta al siguiente problema de investigación: ¿qué procesos de aprendizaje se dan 
en los alumnos al trabajar un AIE sobre el conflicto y la convivencia con una metodología investigativa? Nuestra investigación, reconociendo el carácter contextual del aprendizaje, ha tomado, pues, la forma de una experimentación curricular con seguimiento investigativo realizada en el contexto educativo; se diferencia de muchos estudios sobre concepciones de los alumnos, que suelen seguir el formato de la entrevista clínica de raigambre piagetiana.

El enfoque cualitativo adoptado permite profundizar en la comprensión de los procesos de aprendizaje que se dan en un aula cuando se desarrolla una docencia diseñada para trabajar en torno a problemas sociales relevantes. En todo caso, esto no ha sido obstáculo para el uso de algunos análisis cuantitativos de los resultados cuando se ha considerado necesario. Nos situamos así en una perspectiva plurimetodológica que caracteriza muchas de las investigaciones en educación (Cohen, Manion \& Morrison, 2011).

Con respecto a las fuentes, técnicas e instrumentos para la obtención de los datos, hemos registrado las dinámicas e interacciones en el aula mediante un proceso de observación participante, de larga tradición sobre todo en estudios etnográficos (Cohen, Manion \& Morrison, 2011). Las fuentes utilizadas para ello han sido: los cuadernos de clase de los alumnos (que denominamos como F1, es decir, Fuente 1), las grabaciones en audio de uno de los grupos de debate (F2), seleccionado por sus características, y el diario del investigador (F3). En el desarrollo del proyecto, hemos considerado las tres unidades didácticas (que denominamos U1, U2 y U3) del proyecto educativo experimentadas en el aula como otros tantos "momentos metodológicos" para la recogida de los datos. En cada una de ellas, hemos seleccionado aquellas actividades didácticas que considerábamos con potencialidad para ofrecer informaciones relevantes para la investigación.

Los datos obtenidos de los documentos escritos y de las transcripciones de audio han sido tratados según técnicas de "análisis del contenido" (Cohen, Manion \& Morrison, 2011; Krippendorff, 1990) para ser codificados y convertidos en "unidades de información" utilizando un sistema de categorías, que en este caso hemos centrado — como se ha dicho- en la categoría referida a los aprendizajes conceptuales ("Aprendizajes en la Dimensión Conceptual", en siglas ADC). En esta categoría, tenemos en cuenta varios aspectos: las causas y las consecuencias de los conflictos: el conflicto como proceso; los roles, los contextos y los comportamientos sociales; y las escalas del conflicto. Para esta categoría (ADC) se contemplan, así mismo, tres valores (o formulaciones del conocimiento), siguiendo una hipótesis de progresión de menor a mayor complejidad conceptual (García-Pérez, 2000) (tabla 1). Esta hipótesis de progresión es coherente, por lo demás, con el modelo piagetiano de cambio de las visiones del mundo, que evoluciona desde una posición más egocéntrica y simplificadora hacia una mayor objetivación y abstracción en la concepción de los fenómenos sociales.

Tabla 1

Valores progresivos de la categoría Aprendizajes en la Dimensión Conceptual (ADC)

\begin{tabular}{|c|c|c|}
\hline Valor ADC1 & Valor ADC2 & Valor ADC3 \\
\hline $\begin{array}{l}\text { - La descripción de los fenómenos } \\
\text { sociales está centrada en lo evidente, } \\
\text { con pocos elementos y relacionados } \\
\text { de manera aditiva. } \\
\text { - La caracterización del } \\
\text { comportamiento individual y } \\
\text { colectivo se basa en la perspectiva } \\
\text { de una conducta consciente, } \\
\text { voluntaria y deliberada de los } \\
\text { grandes protagonistas y por la } \\
\text { personalización de las relaciones } \\
\text { entre colectividades. } \\
\text { - Pensamiento precausal y creencia en } \\
\text { el poder del azar y del destino. }\end{array}$ & $\begin{array}{l}\text { - Se manejan conceptos de transición, } \\
\text { como el de "rol social" o el de } \\
\text { "institución social", que acercan la } \\
\text { perspectiva individual a la abstracción de } \\
\text { lo colectivo. } \\
\text { - La caracterización de la conducta } \\
\text { humana gana en matices y se empiezan } \\
\text { a contemplar elementos no perceptibles } \\
\text { y evidentes. } \\
\text { - Se establecen conexiones simples y } \\
\text { lineales entre un factor causal y las } \\
\text { consecuencias de un conflicto. }\end{array}$ & $\begin{array}{l}\text { - Caracteriza la conducta humana } \\
\text { teniendo en cuenta las dicotomías } \\
\text { entre racionalidad e irracionalidad. } \\
\text { Esta perspectiva incorpora la } \\
\text { interacción y la recursividad entre } \\
\text { causas y consecuencias y cómo } \\
\text { estas pueden convertirse a su vez en } \\
\text { factores causales. Tiene en cuenta el } \\
\text { carácter procesual de los fenómenos } \\
\text { sociales. } \\
\text { - Los fenómenos sociales como un } \\
\text { proceso complejo que implica } \\
\text { luchas de intereses y de poder que } \\
\text { conforman intereses y voluntades } \\
\text { colectivas por medio de procesos de } \\
\text { institucionalización de las conductas } \\
\text { y voluntades individuales. }\end{array}$ \\
\hline
\end{tabular}

Fuente: elaboración propia. $A D C=$ Aprendizajes en la Dimensión Conceptual 
Las unidades de información que resultaron de la codificación de los datos fueron gestionadas con el programa ATLAS.ti, a fin de obtener una clasificación conforme al sistema de categorías expuesto, para dar así respuesta al problema investigado. Las unidades de información de valor 1, es decir, de un bajo nivel de complejidad, presentaban las características de una "aproximación predominantemente descriptiva de los fenómenos sociales" (ADC1). Los valores sucesivos, en un orden de complejidad creciente, presentaban las características de una "aproximación predominantemente explicativa de los fenómenos sociales" (ADC2) y de una "aproximación predominantemente comprensiva e interpretativa de los fenómenos sociales" (ADC3).

\section{Resultados y discusión}

Según las creencias epistemológicas, compartidas, por lo demás, por muchos profesores, "se sabe" o "no se sabe", se tiene el conocimiento o se está en el error. Esta concepción "de sentido común" se fundamenta en un esquema de pensamiento simplificador basado en oposiciones binarias. Sin embargo, de nuestra investigación se desprende que, cuando trabajamos con un proyecto de enseñanza que organiza los contenidos en forma de problemas y utiliza una metodología investigativa — como es el caso de este estudio - los conocimientos de los alumnos pueden evolucionar de una manera gradual y dinámica, y son susceptibles de ir adquiriendo diferentes formulaciones progresivamente más complejas. Así lo podemos observar en la tabla 2, que muestra las unidades de información -obtenidas de los datos de las diversas fuentes manejadas - relativas a los aprendizajes en la dimensión conceptual, en valores absolutos y porcentuales, en cada uno de los valores de la hipótesis de progresión (ADC1, ADC2, ADC3) y en cada uno de los tres momentos de la intervención didáctica (U1, U2, U3).

Tabla 2

Unidades de información de los aprendizajes conceptuales

\begin{tabular}{l|l|l|l|l|l|l}
\hline Unidades didácticas & \multicolumn{3}{l|}{ Nivel ADC1 } & \multicolumn{2}{l|}{ Nivel ADC2 } & \multicolumn{2}{l}{ Nivel ADC3 } \\
\hline & $\begin{array}{l}\text { Valores } \\
\text { absolutos }\end{array}$ & Porcentajes (\%) & $\begin{array}{l}\text { Valores } \\
\text { absolutos }\end{array}$ & Porcentajes (\%) & $\begin{array}{l}\text { Valores } \\
\text { absolutos }\end{array}$ & Porcentajes (\%) \\
\hline Unidad 1 (momento 1) & 498 & 77,08 & 148 & 22,91 & 0 & 0,00 \\
\hline Unidad 2 (momento 2) & 329 & 53,06 & 270 & 43,54 & 21 & 3,38 \\
\hline Unidad 3 (momento 3) & 71 & 35,32 & 81 & 40,29 & 49 & 24,37 \\
\hline \hline
\end{tabular}

Fuente: elaboración propia

Por lo demás, en el gráfico 1 podemos observar cómo, en el momento inicial (U1), los porcentajes de unidades de información de valor ADC1 -el de menor nivel de complejidad y por tanto, el más cercano al pensamiento simplificador- son predominantes (77,08\%); por otra parte, no encontramos unidades de información de valor $\operatorname{ADC} 3(0,00 \%)$, pues se trata de un nivel de abstracción y complejidad incompatible con el pensamiento cotidiano. Así mismo, podemos observar la evolución de las concepciones de valor ADC2, que aumentan desde la U1 a la U2, pero disminuyen al final de la intervención (U3), pues empiezan a ser sustituidas por unidades de información del máximo valor, ADC3, mientras las del valor más bajo, $A D C 1$, permanecen sin evolucionar en 35,32\%. 
Gráfico 1

Distribución longitudinal de los valores de la categoría $A D C$

Evolución longitudinal de los Aprendizajes Conceptuales (ADC)

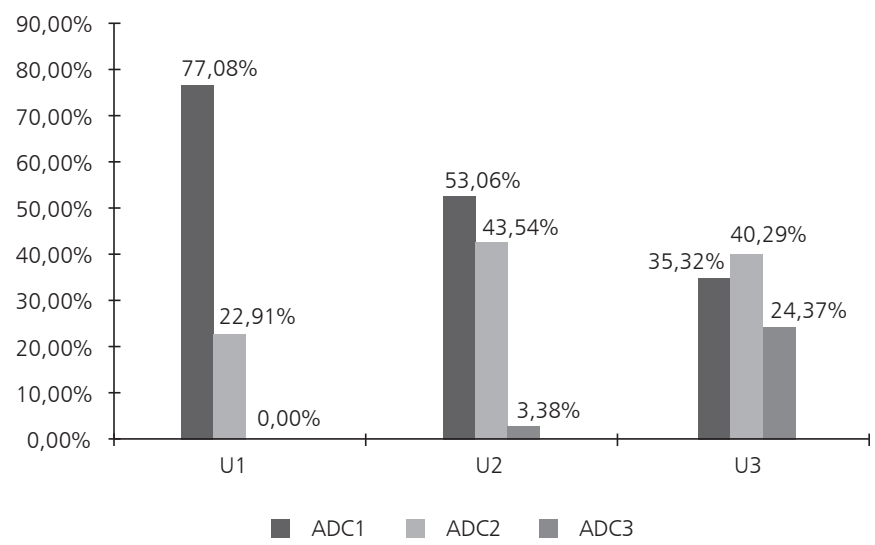

Fuente: elaboración propia

Los resultados de la investigación nos han permitido caracterizar el estado inicial de los conocimientos de nuestros alumnos y alumnas -o "población de ideas" del grupo-clase-, como punto de partida del proceso de enseñanza y aprendizaje, e identificar los obstáculos en la progresión del aprendizaje y los posibles itinerarios didácticos que podrían favorecer este aprendizaje, mediante la selección de contenidos pertinentes y el diseño de actividades adecuadas. Vamos a analizar ahora, con más detalle, las características de las concepciones correspondientes a cada uno de esos valores ( $A D C 1, A D C 2$ y $A D C 3$ ) de nuestra hipótesis de progresión, valores que podemos considerar a modo de niveles de evolución del conocimiento de los alumnos.

Las concepciones de los alumnos en su nivel más bajo de complejidad

Hemos encontrado, en el estado inicial de los conocimientos de nuestros alumnos (ADC1), el predominio de un pensamiento caracterizado por la descripción centrada en lo evidente, que pone en juego elementos escasos, relacionados entre sí de manera aditiva. En esa descripción se destaca la vertiente más perceptible del conflicto, cuyas consecuencias son consideradas normalmente como negativas y relacionadas con el enfrentamiento, la violencia verbal o física y el castigo por parte de alguna figura de autoridad. Es destacable, así mismo, el fuerte tono emocional de las concepciones, lo que dificulta la reflexión al respecto. Con respecto a las causas y las consecuencias de los conflictos, hemos observado que a menudo las relaciones causales no se contemplan, o son inconsistentes; pervive una mentalidad precausal, según la cual los fenómenos ocurren por el azar o el destino. Se puede entrever, también, una cierta "naturalización" de los fenómenos sociales, en el sentido de que no está clara la responsabilidad o la implicación de la acción humana en los hechos (Lenzi \& Castorina, 2000, entre otros):

[¿Cuáles son las causas del conflicto?]: "Alumno 2: porque como estaban en ese lugar en ese momento, pues como tú estás ahí, pues tú pringas, es la ley de la naturaleza" [grabación de grupo de debate]. 
Cuando se contemplan relaciones causales, estas son simples y lineales, y solo se refieren a las causas y consecuencias evidentes y cercanas en el tiempo y en el espacio. Los conflictos y la convivencia dependen de contingencias inmediatas y perceptibles, y no se tienen en cuenta, o se subestiman, la dimensión de las relaciones entre las personas, los factores de la comunicación, la relación previa entre las partes implicadas y la capacidad para tener en cuenta al otro, para dialogar y negociar intereses. El conflicto se "cosifica" y se insiste en los factores materiales implicados, pero al cabo no es una cuestión de relación entre personas sino de contingencias inevitables y un poco azarosas, de mala suerte. Como se recoge en esta unidad de información proveniente de una actividad consistente en el análisis de un conflicto:

[¿Cuáles son las causas de este conflicto, qué factores están implicados?] "Tenemos un incidente en el que se ha inculpado a unas personas por haber estado en el lugar equivocado y en el momento equivocado (...)" [grabación de grupo de debate].

En cuanto a los diferentes cursos o desarrollos que puede tomar un conflicto, por lo general, nuestros alumnos, en este momento de desarrollo de sus concepciones, no entienden el conflicto como un proceso; en todo caso solo conciben una conexión simple, que es más bien una contigüidad de fenómenos, con consecuencias siempre negativas y sin posibilidad de vislumbrar caminos de resolución. A menudo, las consecuencias están relacionadas con el enfado, la violencia verbal o física y el castigo por parte de una figura de autoridad. El desencuentro, la desobediencia y el castigo forman parte del imaginario del conflicto generacional:

[A la cuestión de las posibles consecuencias de un conflicto entre compañeros] "Que se pueden pelear y llegar a las manos, o que el profesor los eche por molestar" [grabación de grupo de debate].

Aunque nuestros alumnos empiezan a utilizar el concepto de rol o función social, no conciben, en esta fase, distintas posibilidades en su ejercicio; el comportamiento viene determinado por el rol que, a su vez, queda asimilado a la posición o cargo que se ocupa en una organización (Lenzi \& Castorina, 2000). Tampoco se distinguen los factores contextuales condicionantes en el desempeño de los roles o funciones sociales. Y, cuando se conciben ambos factores, los contextos y los roles o las funciones sociales obedecen a una lógica determinista: el rol o el contexto determinan el comportamiento. Así, en una actividad consistente en el análisis de un conflicto entre profesores y alumnos por la rotura de un extintor en el que, en ausencia de responsables, se aplicaba un castigo colectivo, no se piensa en la figura del profesor como alguien que medie, que favorezca el diálogo y la resolución negociada de los conflictos mediante la palabra, sino exclusivamente como un vigilante que castiga e impide el desorden y la escalada del conflicto:

[¿Crees que la jefa de estudios y la directora se comportarían igual si no lo fueran? Explícalo] "No, porque ellos son la autoridad en el instituto y tienen que mantener el orden y hacer lo que sea mejor para el instituto" [grabación de grupo de debate].

La caracterización de la conducta en situaciones de conflicto es, pues, muy simplificadora y se ignora la complejidad de la conducta humana. Cuando planteamos a nuestros alumnos una actividad en la que 
debían analizar las conductas de los protagonistas de un conflicto nuclear, y los situamos ante el dilema acerca del predominio de las máquinas en la gestión de la información, en detrimento de la decisión humana, observamos la prevalencia de una visión mecanicista que concibe la duda como una debilidad y la decisión automática de las máquinas como una virtud, lo cual minusvalora la capacidad de control y decisión de las personas:

[¿Son superiores las máquinas en la guerra nuclear?] "Son superiores porque no van a dudar en soltar los misiles" [grabación de grupo de debate].

Las concepciones de nuestros alumnos sobre el conflicto y la convivencia a menudo no distinguen la escala individual y la social; las causas y las consecuencias de los conflictos individuales y de los conflictos sociales observan un gran paralelismo, y se confunden o se solapan las preocupaciones personales y las sociales; es frecuente que los intereses sociales solo se conciban en clave de éxito personal futuro. Esto indicaría un déficit conceptual y un egocentrismo epistemológico que, aunque es característico de etapas anteriores del desarrollo personal, aún subsiste (Delval, 1994), y se expresa con una concepción autorreferencial de los fenómenos sociales. Esto se sustancia en una tendencia a trasladar los esquemas cognitivos implicados en la concepción del conflicto interpersonal a la explicación de los fenómenos sociales. El conflicto social se concibe a semejanza del conflicto individual, como un enfrentamiento entre personas o bien entre grandes protagonistas de los acontecimientos. A menudo, este antagonismo toma la forma de un torneo o enfrentamiento especular entre rivales:

[¿Cuáles eran las causas de los conflictos de la Guerra Fría?] "Pueden empezar por debates unos contra otros y si en el desarrollo de la rivalidad abunda el odio, pueden llegar a guerras nucleares" [grabación de grupo de debate].

Son numerosos los estudios que han mostrado evidencias de este fenómeno. Concretamente, la concepción de la autoridad política "personalizada" funciona como una "representación social" y como obstáculo para la comprensión de lo social (Castorina, Barreiro \& Toscano, 2005). La personalización es, en efecto, un rasgo del conocimiento de los alumnos identificado en diversos trabajos; se trata del rol decisivo que juegan los individuos y las intenciones en la explicación de los hechos históricos y en la comprensión del conocimiento vinculado a lo político, al gobierno nacional, la monarquía, la democracia, etc. (Carretero, Jacott, Limón, López-Manjón \& León, 1994). Desde la perspectiva de las "representaciones sociales", la personalización de los hechos sociales e históricos se interpreta como una apropiación por parte de los sujetos de creencias que preexisten y que se han constituido en la comunicación social, que incluyen la afectividad y los valores, que se dan en todas las edades y son muy resistentes a ser modificadas en los procesos de enseñanza. Sin embargo, según José Antonio Castorina (2005), la presencia de creencias de sentido común no se contrapone con la actividad reconstructiva de los objetos sociales que hacen los sujetos a partir de aquellas representaciones, ni con la originalidad de las nociones alcanzadas en el esfuerzo intelectual.

Otros estudios han puesto de manifiesto la perspectiva personalizadora de las relaciones jerárquicas que muestra en los niños una concepción represiva del jefe como persona que manda, que dice lo que hay que hacer, y una evolución hacia una visión más positiva, paternalista —en los trabajos de Alicia Lenzi y José Antonio Castorina (1999/2000) se denomina a esto "hipótesis benefactora"-, institucional y despersonalizada, que en ocasiones tiene en cuenta el status dentro de una organización.

Todas estas conclusiones indican un predominio, desde la infancia, de la personalización en la concepción de las funciones políticas, que algunos autores denominan "teoría ingenua" o "minimalista", y que persiste hasta la adolescencia tardía; incluso, algunas creencias son muy resistentes a la enseñanza escolar, hasta el punto de que las mantienen algunos adultos legos (Berti, 2005; Delval, 1994). Estas hipótesis se pueden relacionar con creencias básicas como estas: la sociedad está constituida por relaciones individuales y personales no institucionalizadas; los actos de autoridad son personalizados y responden a un jefe máximo; algunos individuos protegen a otros, es decir, unos tienen autoridad sobre otros o los cuidan; el mundo social implica una armonía sin conflicto entre intereses colectivos; los conflictos sociales se reducen a conflictos entre individuos; las relaciones sociales aparecen naturalizadas ya que suceden en un orden independiente de la intervención humana (Lenzi \& Castorina, 2000). Estas creencias son prevalentes en las prácticas sociales, se dan en todas las edades y se muestran resistentes a ser modificadas en los procesos de enseñanza; por lo cual, para los citados autores, parecen reunir todas las características de una representación social.

Los trabajos de Alicia Lenzi y José Antonio Castorina (1999/2000 y 2000) sobre la autoridad escolar y política ponen, así mismo, de manifiesto que los niños construyen hipótesis organizadoras, y a partir de los 12 años aparece lo que denominan "teoría maximalista", según la cual la jerarquía, la legitimidad y los límites de la autoridad dentro de la escuela se explican por 
la hipótesis del "cargo". Por tanto, se dan entidades observables y otras "teóricas", como "cargo", "funciones" o "normas". La elaboración de estas teorías está condicionada por la transmisión de representaciones sociales sobre el mundo político y escolar y por la intervención disciplinante de la autoridad escolar sobre los niños (Castorina, Faigenbaum, Zerbino, Kohen, Tabbush \& Clemente, 2001).

Por tanto, la progresión hacia una objetivación de la institución del gobierno nacional se produce en sucesivas reestructuraciones, en las que las transiciones significativas mantienen cierto paralelismo con la sociogénesis histórica de la institución gubernamental: desde una sostenida personalización de las autoridades gubernamentales hacia su institucionalización y objetivación con la aparición de cargos y funciones normativizadas. En estas transiciones, la diferenciación entre las personas y las funciones sociales que desempeñan es clave; de hecho, para Juan Delval (1994), el objetivo de la enseñanza de lo social es llegar a concebir a las autoridades gubernamentales, no como personas, sino como representantes institucionales, y por tanto, sus actuaciones se hallan institucionalizadas mediante cargos preexistentes establecidos en normas constitucionales que las regulan.

En el caso de la comprensión de un fenómeno histórico como la "Guerra Fría", podemos observar este mecanismo, pues nuestros alumnos — como también ocurre en el pensamiento cotidiano habitual— entienden el enfrentamiento de bloques militares como una lucha entre líderes, como una mera oposición de contrincantes. Cuando se pronuncian sobre el conflicto social e histórico, empiezan a aparecer conceptos aparentemente complejos, pero una mirada más atenta desvela que no lo son tanto. En efecto, cuando se enfrentan a contenidos "académicos" o "escolares" tienden a la simulación del aprendizaje al repetir ideas que se han manejado en las actividades de clase, pero en las que subyace una lógica de pensamiento simplificador (una "pelea de buenos y malos"). Según algunos autores (por ejemplo, Delval, 1994), los alumnos establecen dos tipos de explicaciones, no conectadas: una para comprender los fenómenos de la vida cotidiana y otra para la actividad en la escuela, y mantienen así dos contextos del conocimiento que apenas interaccionan entre sí. En nuestro caso, apreciamos que bajo la superficial "comprensión" académica subyace la lógica simplificadora, de sentido común, del pensamiento cotidiano, pues no se manejan conceptos mediadores o de transición entre lo individual y lo colectivo. Así, las causas del conflicto social siguen remitiendo a enfrentamientos y rivalidades, y las consecuencias siguen siendo las elementales y perceptibles, la enemistad o la violencia:

[Al pronunciarse sobre una situación, fabulada, en el Instituto] "Esto es lo que ha pasado en el instituto, un conflicto (...) salieron muchos heridos y también lo que ocurrió hace muchos años en las guerras entre los países como fueron la primera y la segunda guerra mundial (...) fueron causadas por hombres que opinaban diferente" [grabación de grupo de debate].

Desde una perspectiva didáctica, esta interpretación nos ayuda a entender lo difícil que es desarraigar o desbloquear estas concepciones que están alimentadas por la ideología dominante, frente a las que la escuela no siempre tiene suficientes armas para luchar. El trabajo en torno a problemas podría ser una alternativa válida para modificar, por medio de la investigación escolar, estas creencias socialmente instaladas y construir progresivamente, con argumentos e informaciones más potentes, nuevas ideas. 
Avances en la progresión de los aprendizajes

Frente a la visión simplificadora que se acaba de exponer, ahora en este valor de las concepciones (ADC2) las causas y las consecuencias de los conflictos empiezan a tener vinculación con las personas y con sus características, o con fenómenos complejos de comunicación entre las personas. En el caso propuesto de análisis de un conflicto ocurrido en el centro escolar, en el que, en ausencia de un responsable, se aplicaba un castigo colectivo ejemplarizante, ahora no se esgrimen como causas las contingencias de espacio o tiempo - estar en el lugar equivocado en el momento equivocado-, sino que se apunta directamente a un conflicto entre personas con poderes desiguales:

"Alumno 1: pero que la causa del conflicto es que les echan la culpa a ellos (...).

Alumno 3: vamos a ver, que han roto un extintor y le echan la culpa a $4^{\circ}$ de ESO sin saber si han sido ellos.

Alumno 4: y no tienen pruebas para acusarlos" [grabación de grupo de debate].

Interpretamos estas aportaciones como un progreso en la construcción de la idea de relaciones de poder y de conflicto generacional, tan importante para comprender las relaciones complejas de convivencia con los adultos en las que están inmersos nuestros alumnos. En cuanto a las causas de los conflictos interpersonales, y en un orden de complejidad creciente, se describen ya distintos factores, como la falta de empatía, la intolerancia y el impulso de dominación, que conducen a la satisfacción de las propias necesidades y deseos sin contar con la otra parte. Además, junto a causas y consecuencias perceptibles aparecen otras menos evidentes, y factores inferidos de la situación o diferidos en el tiempo y en el espacio. Así lo vemos en una actividad consistente en el análisis de los problemas complejos de convivencia que se dan en la película American History $X$.

"Las causas son que el hijo sigue los mismos pasos que el padre. El conflicto racial también es provocado por las desconfianzas entre personas de distinto color. También puede crearlo por las desigualdades que hay en cada país" [cuaderno de alumno].

El miedo y la desconfianza se conciben como factores acumulativos o agravantes del conflicto. En el proceso se distinguen distintas fases o momentos en los que un conflicto que no se arregla puede permanecer larvado u oculto, condicionar las relaciones futuras o desencadenarse con posterioridad. Este matiz se refiere al hecho de que la represión del conflicto no lo soluciona, sino que lo agrava, pues impide su expresión:

"El miedo y la desconfianza aumentan el conflicto porque (...) puede estar larvado o latente (solucionado sin llegar a solucionarlo realmente) y más tarde se puede reanudar con más violencia y agresividad" [cuaderno de alumno].

En el caso de conflictos sociales, como las guerras, las estrategias pasan por tratados de paz que impliquen verdadera reconciliación, "pues de lo contrario no habrá auténtica resolución". Esto lleva a nuestros alumnos a postular que, en el caso de un "mal arreglo" — se pone como ejemplo el Tratado de Versalles de 1919-, los problemas no se solucionan, sino que se agravan, pues el conflicto permanece latente y condiciona las relaciones 
futuras. Esto supone una salida del imaginario de la simplificación y de la prevalencia de lo aparente y un paso hacia un pensamiento más complejo que hace posible una actitud más favorable hacia la gestión de los conflictos. Así se aprecia también en la idea de "escalada del conflicto", por lo que supone la comprensión de un proceso que puede conducir a la construcción del concepto de "interacción":

"El conflicto va escalando para peor (...) la desconfianza puede alimentarse de varios factores, puede formar un círculo vicioso en el que la desconfianza provoca desconfianza" [cuaderno de alumno].

Con respecto a la concepción de los "roles y funciones sociales", la progresión observada consiste en el paso del determinismo al posibilismo. Es decir, los roles y los contextos condicionan la conducta pero no la determinan, los distintos estilos personales también influyen. En el caso del conflicto en el centro escolar por la rotura de un extintor observamos:

"El profesor puede mirar por sus alumnos (...) si él cree que no ha sido el grupo, tiene que defenderlos, él los puede defender" [grabación de grupo de debate].

Eso supone una visión más compleja de la conducta en las organizaciones y distinguir el contenido manifiesto del contenido latente de un discurso, trabajar con distintas opciones, interpretar los silencios, inferir la información ausente a partir de un documento o entrevista.

[Valoración de las entrevistas realizadas por los alumnos a algunos profesores]: "Le preguntamos que si podríamos cambiar la organización y dijo que lo teníamos crudo. Yo creo que no sería difícil porque sin alumnos no hay instituto ni nada. Ha asumido su rol de profesor y nos ha infravalorado porque cree que dada nuestra posición, no podemos hacer nada" [cuaderno de alumno].

Por lo demás, tener en cuenta los roles y caracterizar las conductas permite realizar pequeños análisis comparativos a distintas escalas:

"Si un hombre como Cameron [Alexander, personaje de la película American History $X$ ] llega al gobierno, podría actuar como [Adolfo] Hitler (...), si las bandas neonazis organizadas llegasen a imponer sus ideas, las consecuencias pueden ser que vuelva a ocurrir lo que pasó con Hitler o con [Francisco] Franco" [cuaderno de alumno].

Y permite también interpretar los fenómenos históricos teniendo en cuenta las relaciones de poder y cómo estas se institucionalizan:

[¿Se pudo haber evitado lanzar las bombas atómicas sobre Hiroshima y Nagasaki?]: "Sí, porque Japón estaba derrotada y estaba planeando la rendición (...) USA lanzó las bombas solo para demostrar el poder ante otros países (Rusia)" [grabación de grupo de debate].

Hacia metas más complejas de aprendizaje

Esta otra perspectiva (representada por el valor ADC3) incorpora el concepto de interacción y de recursividad entre causas y consecuencias. Esto implica una concepción del conflicto como un proceso complejo en el que son posibles distintas fases y desarrollos. Y en medio de estos se 
contemplan momentos en los que el conflicto puede permanecer larvado o latente, y expresarse con posterioridad activado por algún factor desencadenante:

“(...) un mal arreglo de un conflicto como el de la primera guerra mundial y el tratado de Versalles puede dar lugar a un conflicto mayor como la segunda guerra mundial donde las alianzas son más poderosas (...) también puede observarse en Alemania (...) que aunque el conflicto esté latente puede que condicione una mala relación entre las partes acabando en otro conflicto, esto es otro tipo de consecuencia (...), consecuencia circular, es decir, que nunca se termina de solucionar el conflicto ya que las causas se relacionan entre sí formando un círculo vicioso" [cuaderno de alumno].

La toma de conciencia de las relaciones de poder permite un pensamiento más reflexivo y una mayor capacidad para la inferencia y la interpretación; esto hace posible una comprensión más compleja de los fenómenos humanos y sociales. Así puede verse en este fragmento:

"Alumno 4: yo creo que tenemos muchos derechos pero no se implantan (...) vamos a ver (...) que nosotros tenemos derecho a algo pero no se hace, los profesores no (...).

Alumno 3: que tenemos muchos derechos pero no se llevan a cabo (...).

Alumno 5: yo pienso que tenemos muchos derechos pero la mitad no lo sabemos.

Alumnos 1: cuando le hicimos la entrevista a los de jefatura y le preguntamos por nuestros derechos, no quiso tirar por esa rama (...).

Alumnos 2: en conclusión, sabemos que tenemos derechos pero que en la mayoría de los casos se nos ignora o no se aprecian nuestras ideas" [grabación de grupo de debate].

Con respecto a la concepción de los roles, los contextos y los comportamientos sociales, en este valor ADC3 encontramos una progresiva sustitución de los esquemas cognitivos simplificadores, con respecto a la personalización, por otro tipo de estrategias más complejas que manejan las diferentes escalas y contextos. Así mismo, observamos la utilización de conceptos mediadores entre la fenomenología de lo individual y lo social, como la organización social, el liderazgo, la ideología, las relaciones de poder, etc. Se describe al líder autoritario y los mecanismos de dominación que utiliza, así como las consecuencias que tiene en términos de pérdida de capacidad de decisión de sus seguidores. Es decir, se produce una transición entre la fenomenología de las relaciones interpersonales y los hechos colectivos que permite una mejor comprensión de lo social. En situaciones de conflicto social se describen estas conductas y las consecuencias sociales: la dictadura y/o la guerra, como podemos observar en una actividad consistente en el análisis de los distintos tipos de liderazgo que aparecen en las películas Marea roja y $\sin$ novedad en el frente:

[¿Qué características tiene un líder autoritario?, ¿Qué consecuencias tiene? ¿Qué relación se establece entre el líder autoritario y sus seguidores?]: "(...) en mi opinión que sea dominante en algunos aspectos, como en Marea roja [la película]; el comandante [Frank] Ramsey era arrogante (...) estas personas empiezan siendo egocéntricos, suelen ser severos, duros, exigentes, les fascina el poder y la fuerza y desconfían de las personas (...) van consiguiendo poco a poco a más personas que piensen como él sobre temas, creando así grupos cada vez más poderosos (...) las consecuencias de tener un líder autoritario pueden ser que un país pueda acabar sometido a las leyes de un dictador" [cuaderno de alumno].

Entre los distintos estilos de liderazgo, el concepto de "liderazgo mesiánico" supone un mayor ejercicio de abstracción, pues implica una relación dialéctica entre los fenómenos individuales - las características y los comportamientos del líder-y los fenómenos sociales - las demandas colectivas- La crisis social se describe en términos de sentimientos, actitudes y conductas de la ciudadanía que demanda la seguridad de un líder que promete un futuro mejor. Las características de personalidad del líder se entienden como la respuesta a una demanda social en una coyuntura social, política, económica y de mentalidad determinada. De esta forma, los factores económicos y políticos se conectan con los sociales y de mentalidad colectiva, como podemos observar en una actividad consistente en el análisis de los liderazgos en los regímenes fascistas:

"(...) la misión de un líder mesiánico es llegar al poder a toda costa, se cree un elegido o enviado (sus adeptos aceptan cualquier sacrificio incluso la muerte), también tiene como misión de dominar a las personas (...) utiliza la amenaza y la promesa como instrumentos de dominación (...), utiliza el miedo, la inseguridad y el odio en su beneficio (...) a veces lo provoca para ofrecer sus soluciones (...) lo ven como un elegido o enviado para que los protejan y que tengan esperanza y fe, les hace creer que es un mesías, un salvador (...)" [cuaderno de alumno].

Esta complejización del pensamiento de los alumnos permite aplicar los conceptos aprendidos en el marco de la investigación histórica, realizada en 
clase, a la reflexión sobre los fenómenos del presente. Así, en una actividad consistente en el análisis de los movimientos sociales actuales, se plantean algunos dilemas:

"Alumno 4: ¿y el poder de los líderes y de los partidos, qué crees?

Alumno 1: yo opino que no hace falta tener líder (...) ni tener un partido ni nada de eso si se ve que todo el mundo está a favor del $15 \mathrm{M}$ [el movimiento popular ocurrido en España contra la política convencional a partir del 15 de mayo de 2011] (...) tienen el pueblo en contra.

Alumno 5: yo creo que sin un líder y sin un partido no van a conseguir nada porque primero se tienen que organizar y ya después (...).

Alumno 4: entonces sería lo mismo, ino?, porque cuando (...) porque si tienen un líder y llega al poder icómo sabemos que no va a hacer igual que los otros?" [grabación de grupo de debate].

\section{Conclusiones}

De lo expuesto en los resultados de esta investigación se desprende la existencia de una cierta dualidad en las manifestaciones de las concepciones o representaciones de los alumnos. Por una parte, su actividad de construcción del conocimiento se halla restringida por un trasfondo de creencias y concepciones que tienen su origen en el contexto académico; ello da lugar a un conocimiento que no refleja la lógica del pensamiento de estos alumnos, sino que se orienta, básicamente, a satisfacer los requerimientos de la escuela y del profesorado. Por otra parte, las representaciones que tienen su origen en el contexto social y en el pensamiento de sentido común, provenientes del medio familiar o de la interacción entre iguales, son, en general, menos fingidas y reflejan mejor la lógica profunda del pensamiento de estos alumnos. Pero estas ideas de sentido común, menos "contaminadas" por el marco escolar, son igualmente simplificadoras. En todo caso, estas concepciones del alumnado - tengan un origen u otro- son susceptibles de ser modificadas y enriquecidas por propuestas didácticas que, teniéndolas en cuenta, ofrezcan posibles itinerarios de progresión del conocimiento, si bien habría que trabajar didácticamente para desbloquear las restricciones antes citadas.

En ese sentido, sería necesario continuar investigando, desde el campo de la didáctica, sobre cómo favorecer la deconstrucción de la cultura escolar academicista presente en el alumnado, para poner en primer plano sus concepciones de sentido común y favorecer, a partir de ellas, la construcción de visiones más complejas del mundo social. Algunas evidencias apuntan a que esto se puede favorecer cuando se trabajan contenidos que conectan con problemáticas relevantes y sentidas por los alumnos, y se respeta su protagonismo utilizando una metodología didáctica investigativa, acorde con el tratamiento de los problemas estudiados. Consideramos así —en la línea de otras investigaciones realizadas en el marco del proyecto IRES - que el "modelo didáctico de investigación en la escuela" (García-Pérez, 2000) contribuye, en la práctica, al aprendizaje de los alumnos, al favorecer el enriquecimiento progresivo de sus concepciones hacia metas de conocimiento más complejo y deseable.

Más concretamente, en nuestra propuesta didáctica de AIE sobre el conflicto y la convivencia, la progresión conceptual pasó primero por la identificación de las personas como el factor clave de la convivencia, para a continuación profundizar en la caracterización compleja de su conducta y de sus relaciones, especialmente las no visibles, como articuladoras de esa convivencia: los conflictos de intereses y las relaciones de poder. Pero la personalización de los fenómenos sociales toma como modelo el comportamiento de las personas para explicar los hechos sociales, y supone un obstáculo para comprenderlos. En este sentido, la introducción del concepto de rol o función social, y específicamente el concepto de liderazgo social, supuso un paso decisivo en la comprensión de la idea, más abstracta, de "sociedad".

Concluimos que, desde un punto de vista didáctico, ha resultado útil trabajar estos conceptos en la frontera entre lo individual y lo social, pues ello facilita la comprensión de la convivencia cercana y del papel de los líderes y sus implicaciones a escala colectiva. Por lo demás, los resultados obtenidos al respecto confirman conclusiones anteriores de nuestro marco de investigación, sobre la importancia de la utilización de una gradación conceptual adecuada en las propuestas didácticas, y más específicamente sobre el uso, en momentos clave del proceso, de conceptos mediadores que puedan ayudar a superar determinados umbrales de dificultad en los aprendizajes.

Estamos conscientes del carácter limitado de estas conclusiones, pues se ha indagado solo sobre la dimensión conceptual de las concepciones, si bien este estudio se enmarca en una línea de investigación más amplia que ha obtenido también resultados sobre las dimensiones procedimentales y actitudinales del conocimiento, y abre, así mismo, el camino a investigaciones que sigan profundizando en la superación de la lógica de la cultura escolar dominante.

Por lo demás, al tratarse de una investigación realizada con metodología cualitativa, los resultados ayudan, básicamente, a comprender los procesos de construcción del conocimiento a partir de la muestra analizada en el estudio de caso, sin que se pretenda 
una generalización de estas conclusiones. En todo caso, consideramos que estas aportaciones son relevantes y permiten confirmar la potencialidad de la metodología didáctica (de trabajo en torno a problemas) aplicada para la enseñanza de las ciencias sociales en el aula, por favorecer un enriquecimiento progresivo del conocimiento cotidiano del alumnado y por adecuarse al carácter complejo de los propios fenómenos sociales.

\section{Sobre los autores}

José Antonio Pineda-Alfonso ha desarrollado en los últimos años su docencia en el campo de la formación de maestros y profesores de secundaria y su investigación en el campo de la didáctica de la historia, la educación para la ciudadanía y la participación, la convivencia escolar y la formación del profesorado.

Francisco F. García-Pérez es autor de diversos libros, capítulos de libros y artículos de educación. Ha participado en diversas investigaciones sobre innovaciones educativas y sobre formación del profesorado. Director de diversas tesis doctorales, actualmente centra su investigación en la enseñanza de la participación ciudadana y en la formación del profesorado.

\section{Referencias}

Álvarez, Leonardo Yovany (2012). Representaciones sobre el gobierno en niños, niñas y adolescentes de Bucaramanga. Revista Latinoamericana de Ciencias Sociales, Niñez y Juventud, 10 (2), 883-896. Disponible en: http://www.scielo.org.co/pdf/rlcs/v10n2/v10n2a08.pdf

Barrett, Martyn D. \& Buchanan-Barrow, Eithne (2002). Children's Understanding of Society. En Peter K. Smith \& Craig H. Hart (eds.). Blackwell Handbook of Childhood Social Development, 491-512. Malden, Massachusetts: Blackwell.

Berti, Anna Emilia (2005). Children's Understanding of Politics. En Martyn D. Barrett \& Eithne Buchanan-Barrow (eds.). Children's Understanding of Society (Studies in Developmental Psychology), 69-104. Hove, United Kingdom: Psychology Press.

Carretero, Mario; Jacott, Liliana; Limón, Margarita; López-Manjón, Asunción \& León, José A. (1994). Chapter 15, Historical Knowledge: Cognitive and Instructional Implications. En Mario Carretero \& James F. Voss (eds.). Cognitive and Instructional Processes in History and the Social Sciences, 357-376. Hillsdale, New Jersey: Lawrence Erlbaum.

Castorina, José Antonio (2005). La investigación psicológica de los conocimientos sociales. Los desafíos a la tradición constructivista. En José Antonio Castorina (coord.). Construcción conceptual y representaciones sociales. El conocimiento de la sociedad, 19-40. Buenos Aires: Miño y Dávila.

Castorina, José Antonio; Barreiro, Alicia \& Toscano, Ana Gracia (2005). Dos versiones del sentido común: las teorías implícitas y las representaciones sociales. En José Antonio Castorina (coord.). Construcción conceptual y representaciones sociales. El conocimiento de la sociedad, 205-238. Buenos Aires: Miño y Dávila.

Castorina, José Antonio; Faigenbaum, Gustavo; Zerbino, Mario; KohenKohen, Raquel; Tabbush, Constanza \& Clemente, Fernando (2001). El conocimiento social de los niños y las prácticas institucionales. Revista Instituto Rosario de Investigación en Ciencias de la Educación, IRICE, 15, 31-54. Disponible en: http://rephip.unr.edu.ar/hand le/2133/4796 
Cohen, Louis; Manion, Lawrence \& Morrison, Keith (2011). Research Methods in Education, $7^{\text {th }}$ ed. New York: Routledge.

Delval, Juan (1994). El desarrollo humano. Madrid: Siglo XXI.

Enesco, lleana; Delval, Juan; Navarro, Alejandra; Villuendas, Dolores; Sierra, Purificación \& Peñaranda, Ana (1995). La comprensión de la organización social en niños y adolescentes. Madrid: Ministerio de Educación y Ciencia, Centro de Investigación y Documentación Educativa, CIDE. Disponible en: https://sede.educacion.gob.es/publiventa/descar ga.action?f_codigo_agc $=1388 \_19$

García-Díaz, José Eduardo (1998). Hacia una teoría alternativa sobre los contenidos escolares. Sevilla: Díada.

García-Pérez, Francisco F. (2000). Un modelo didáctico alternativo para transformar la educación: el modelo de investigación en la escuela. Scripta Nova. Revista Electrónica de Geografía y Ciencias Sociales, IV (64), 55-78 (15 de mayo de 2000). Disponible en: http://www.ub.edu/geocrit/sn-64.htm.

Krippendorff, Klaus (1990). Metodología de análisis de contenido: teoría y práctica. Barcelona: Paidós.

Lenzi, Alicia; Borzi, Sonia; Pataro, Alejandra \& Iglesias, Cristina (2005). La construcción de conocimientos políticos en niños y jóvenes. Un desafío para la educación ciudadana. En José Antonio Castorina (coord.). Construcción conceptual y representaciones sociales. El conocimiento de la sociedad, 71-98. Buenos Aires: Miño y Dávila.

Lenzi, Alicia \& Castorina, José Antonio (1999/2000). El cambio conceptual en conocimientos políticos. Aproximación a un modelo explicativo. Revista Latina de Pensamiento y Lenguaje, 7 (1), 125-144.

Lenzi, Alicia \& Castorina, José Antonio (2000). Algunas reflexiones sobre una investigación psicogenética en conocimientos sociales: la noción de autoridad escolar. En José Antonio Castorina \& Alicia Lenzi (comps.). La formación de los conocimientos sociales en los niños. Investigaciones psicológicas y perspectivas educativas, 41-58. Barcelona: Gedisa.

Lobato-Junior, Antonio (2013). Representaciones sociales y didáctica: construcción teórica de un espacio común. magis, Revista Internacional de Investigación en Educación, 5 (11), 277-295. Disponible en: http://revistas.javeriana.edu.co/ index.php/MAGIS/article/view/5648/4726

Misco, Thomas (2009). "The Children Don't Have Any Idea": How Case Studies of Controversy Can Help Build Democratic Habits of Mind. magis, Revista Internacional de Investigación en Educación, 1 (2),
329-340. Disponible en: http://revistas.javeriana. edu.co/index.php/MAGIS/article/download/33 $88 / 2577$

Moscovici, Serge (2003). La conciencia social y su historia. En José Antonio Castorina (comp.). Representaciones sociales: problemas teóricos y conocimientos infantiles, 91-110. Barcelona: Gedisa.

Overton, Willis F. (2013). New Paradigm for Developmental Science: Relationism and RelationalDevelopmental Systems. Applied Developmental Science, 17 (2), 94-107.

Pineda-Alfonso, José Antonio (2011). Las concepciones de los alumnos sobre el conflicto y la convivencia. Un estudio con alumnado de $4^{\circ}$ de ESO. Investigación en la Escuela, 75, 35-47. Disponible en: https://idus.us.es/xmlui/bitstream/hand le/11441/25045/Las_concepciones_de_los_ alumnos_sobre_el_conflicto_y_la_convivencia. pdf? sequence $=\overline{1}$

Pineda-Alfonso, José Antonio (2013). "El conflicto y la convivencia". Experimentación de un ámbito de investigación escolar y análisis del desarrollo profesional docente. Tesis doctoral. Departamento de Didáctica de las Ciencias Experimentales y Sociales. Facultad de Ciencias de la Educación. Universidad de Sevilla. Disponible en: http:// fondosdigitales.us.es/tesis/tesis/2079/el-con flicto-y-la-convivencia-experimentacion-de-unambito-de-investigacion-escolar-y-analisis-deldesarrollo-profesional-docente/\#description

Pineda-Alfonso, José Antonio \& García-Pérez, Francisco F. (2011). La construcción de un ámbito de investigación escolar sobre el conflicto, la violencia y la guerra. Íber, Didáctica de las Ciencias Sociales, Geografía e Historia, 68, 82-91. Disponible en: https://www.academia.edu/27495878/La_ construcci\%C3\%B3n_de_un_\%C3\%A1mbito_ de_investigaci\%C3\%B3n_escolar_sobre_el_con flicto_la_violencia_y_la_guerra.pdf

Torney-Purta, Judith; Lehmann, Rainer; Oswald, Hans \& Schulz, Wolfram (2001). Citizenship and Education in Twenty-eight Countries. Civic Knowledge and Engagement at Age Fourteen. Amsterdam: The International Association for the Evaluation of Educational Achievement (IEA). Disponible en: http://files.eric.ed.gov/fulltext/ED452116.pdf

Treagust, David F. \& Duit, Reinders (2015). On the Significance of Conceptual Metaphors in Teaching and Learning Science: Commentary on Lancor; Niebert and Gropengiesser; and Fuchs. International Journal of Science Education, 37 (5-6), 958-965. Disponible en: http://dx.doi.org/10.10 80/09500693.2015.1025312 